\title{
ALICE Transition Radiation Detector
}

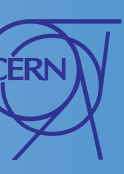

The Transition Radiation Detector (TRD) is the main electron detector in ALICE. In conjunction with the TPC and the ITS, it provides the necessary electron identification capability to study:

- Production of light and heavy vector mesons as well as the continuum in the di-electron channel

- Semi-leptonic decays of hadrons with open charm and open beauty via the single-electron channel using the displaced vertex information provided by the ITS

- Correlated $D \bar{D}$ and $B \bar{B}$ pairs via coincidences of electrons in the central barrel and muons in the forward muon arm

- Jets with high $E_{t}$ by requiring several high $p_{t}$ tracks in one single TRD module.

\section{Principle of Operation}

Each detector module is a large area drift chamber with radiator material in front. A plane of cathode wires separates the drift region with uniform electric field from the amplification region. A charged particle passing through the gas volume creates a trail of ionisation electrons, which drift towards the amplification region. At the anode wires these electrons create avalanches, thereby inducing signals on a segmented cathode plane.
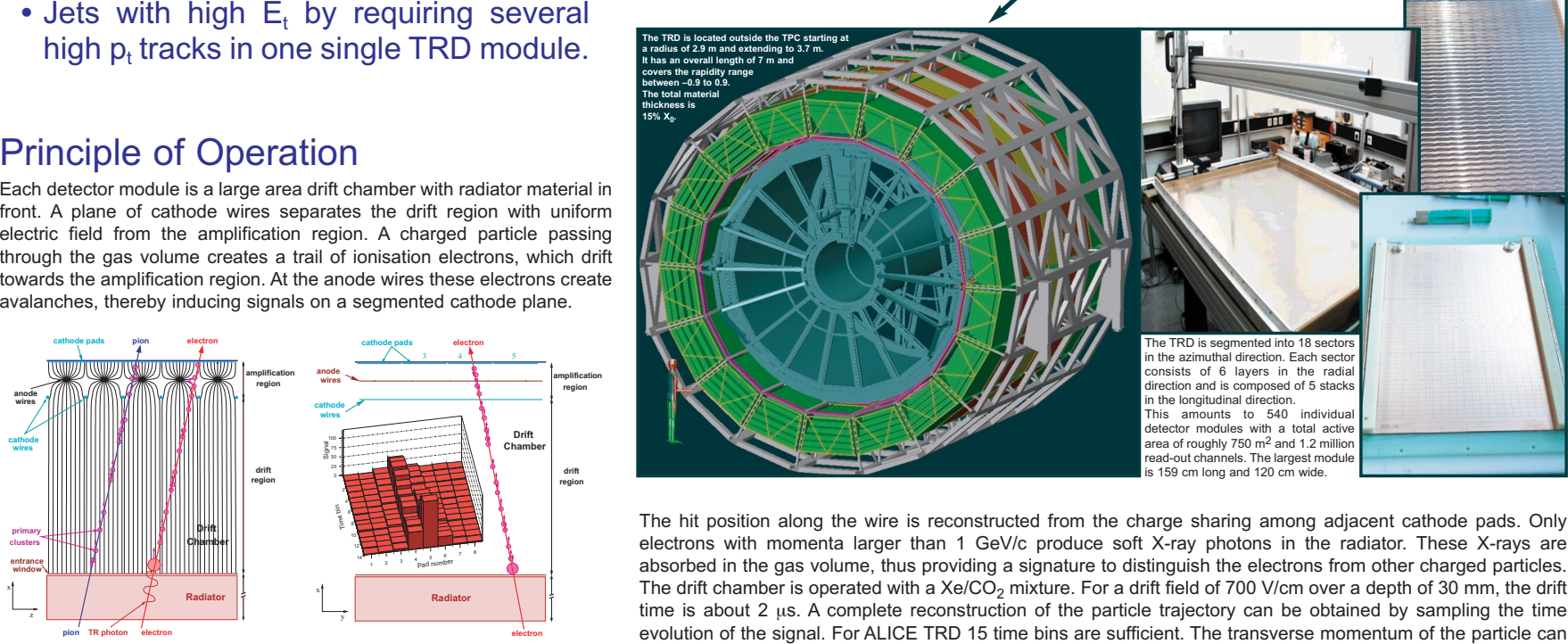

The hit position along the wire is reconstructed from the charge sharing among adjacent cathode pads. Only electrons with momenta larger than $1 \mathrm{GeV} / \mathrm{c}$ produce soft X-ray photons in the radiator. These X-rays are absorbed in the gas volume, thus providing a signature to distinguish the electrons from other charged particles. The drift chamber is operated with a Xe/CO $\mathrm{CO}_{2}$ mixture. For a drift field of $700 \mathrm{~V} / \mathrm{cm}$ over a depth of $30 \mathrm{~mm}$, the drift time is about $2 \mu \mathrm{s}$. A complete reconstruction of the particle trajectory can be obtained by sampling the time evolution of the signal. For ALICE TRD 15 time bins are sufficient. The transverse momentum of the particle can be calculated from the deflection of the trajectory in the magnetic field.

The Radiator
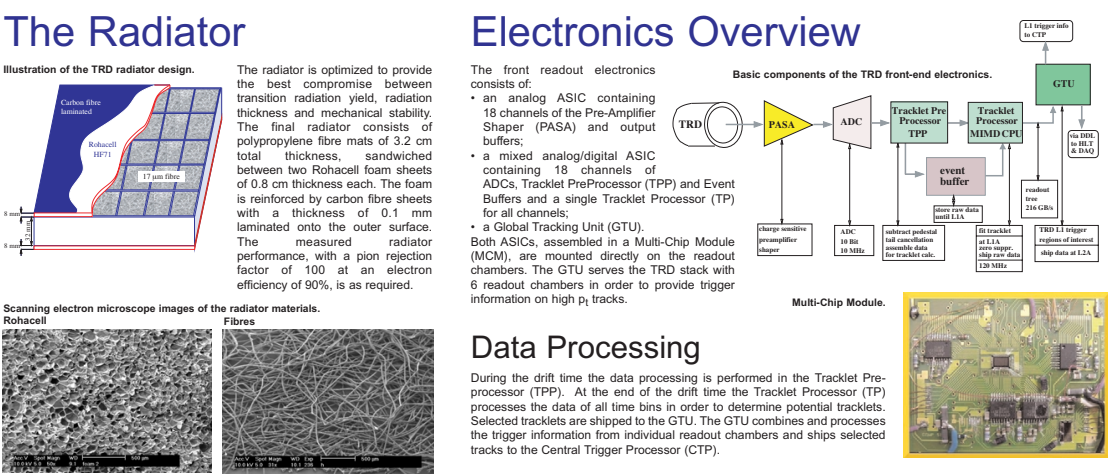

Data Processing

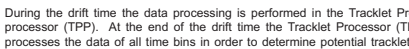

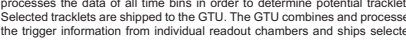

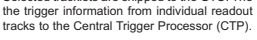

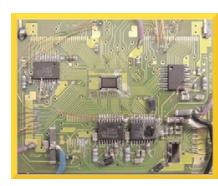

The Trigger Concept

Local Tracking Unit

Tasks of digital chip on detector
select good clusters

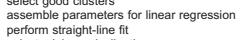

Global Tracking Unit

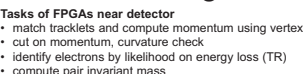

Event Selection
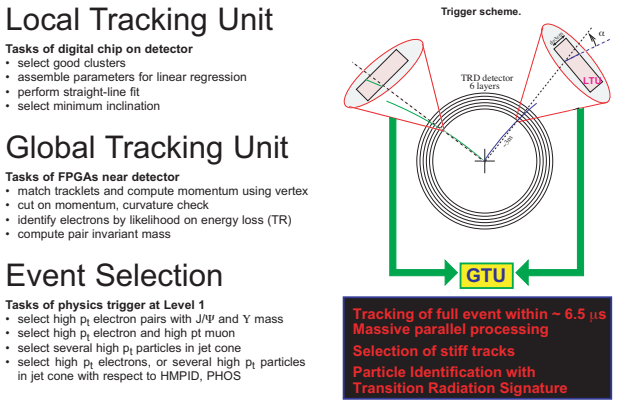

\section{Detector Performance}

Electron Identification

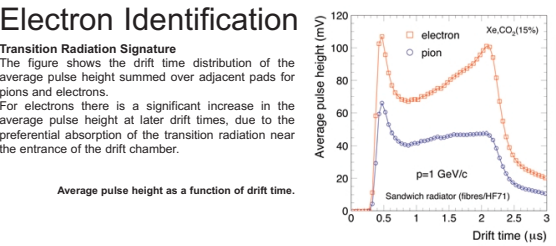

Momentum Resolution

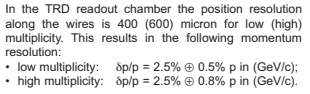
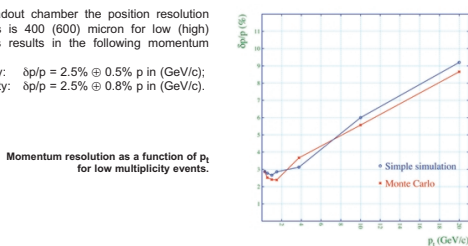

\section{Pion Rejection}
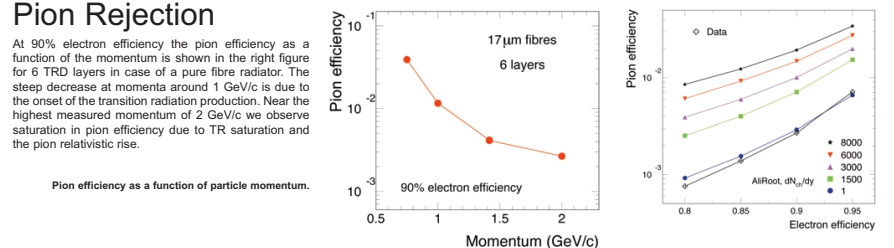

Going from well isolated tracks (data) to full
multipilicity (simulation) we observe deterioration up to a factor of $6-7$. . However, the
rejection factor at $90 \%$ electron efficiency is stil
close to 100 as required.

Pion efficiency as a function of electron efficionn
and the total charged particiele multitipicity.
$\Upsilon$ Invariant Mass Resolution

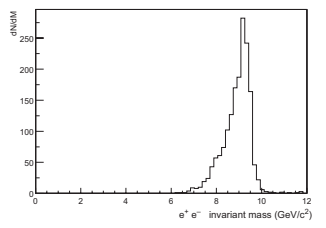

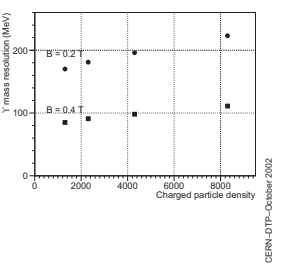

